\title{
Study of the synthesis of a copolymer of isodecyl- and benzylmethacrylates after radiation initiation
}

\author{
Ilya Antonov ${ }^{1}$, Boris Tolochko ${ }^{1,2}$, Alexandr Varand $^{1,2}$, Mikhail Mikhailenko ${ }^{1,2}$, Ilya Eltsov ${ }^{3}$, \\ Alexandr Bryazgin ${ }^{2}$, and Eugene Smirnov ${ }^{4}$ \\ ${ }^{1}$ Institute of Solid State Chemistry and Mechanochemistry SB RAS, Novosibirsk, 630128 Russian \\ Federation \\ ${ }^{2}$ Budker Institute of Nuclear Physics SB RAS, Novosibirsk, 630090 Russian Federation \\ ${ }^{3}$ Novosibirsk State University, Novosibirsk, 630090 Russian Federation \\ ${ }^{4}$ Russian Federal Nuclear Center - Zababakhin All-Russia Research Institute of Technical Physics, \\ Snezhinsk, 456770 Russian Federation
}

\begin{abstract}
Polymerization was carried out using the method of radiation initiation. Experiments were carried out on methacrylic acid derivatives with bulky substituents (isodecyl and benzyl). The regularities of its formation are investigated. The conditions for obtaining a homogeneous copolymer are determined. A polymer with a molecular weight of more than $10^{6} \mathrm{~g} / \mathrm{mol}$ was obtained. An increase in the viscosity of the copolymer with an increase in the temperature of the solution was shown.
\end{abstract}

\section{Introduction}

Poly(methyl methacrylate) (PMMA) has a number of desirable properties, such as outstanding transparency, stability upon severe conditions, high surface resistivity, resistance to weathering and moisture. Due to these superior characteristics PMMA has been widely used as sizer, additive, coating and polishing agents, binder, sealer, transparent neutron stopper, optical fiber and so on. Also PMMA may be used in high voltage application and outdoor electrical application [1]. Won Seok Lyoo et al. [2] reported that high molecular weight (HMW) and superior molecular structure, namely minimum quantity of branches is essential to enhance these PMMA properties. The paper also gives an overview of four MMA polymerization methods (bulk, solution, emulsion, and suspension). It was stated that PMMA HMW could be achieved through radical polymerization, and minimum quantity of branches could be achieved by using suspension-based method.

However, removing the emulsifier and suspension agent from PMMA polymer is complex and costly, and the residual emulsifier and suspension agent in final PMMA products often deteriorate its optical and electronic properties. But using the method of physical initiation agents allows obtaining HMW polymers (bulk, solution) without the use of initiators and inhibitors [3]. The resulting polymers make it possible to synthesize more 
filled composites in comparison with the composites based on high molecular weight polymers obtained by traditional methods.

The aim of this work was to study the possibility of using radiation initiation for the polymerization(Fig. 1) of methacrylic acid esters with large substituents (isodecyl methacrylate, benzyl methacrylate), obtaining their copolymer, and studying the laws of synthesis in order to obtain a homogeneous copolymer.
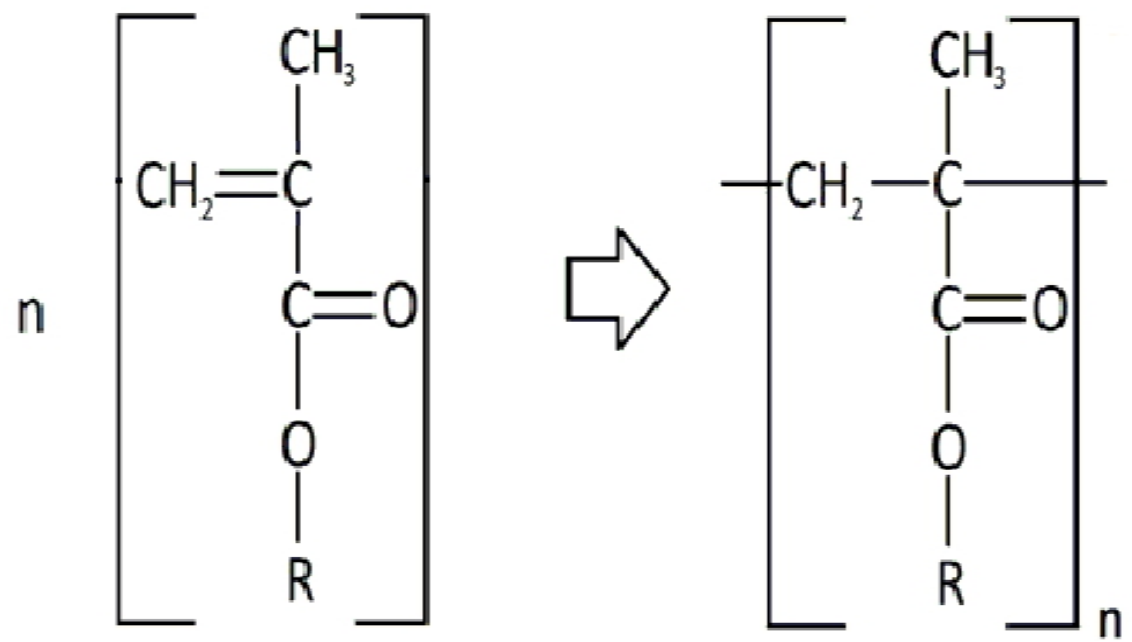

Fig. 1. Structural formula of copolymer $(\mathrm{R}=$-isodecyl and/or -benzyl $)$.

\section{Experimental}

\subsection{Materials}

Ethyl acetate, ethanol, tetrahydrofuran stabilized, isodecyl methacrylate stabilized (Evonik), benzyl methacrylate stabilized (Evonik).

Tetrahydrofuran was purified before use by distillation under reduced pressure using a rotary evaporator. Purification of acrylates was carried out via separation funnel. A volume of distilled water was added to the same volume of acrylate, mixed, and an hour later the aqueous solution was separated from the mixture.

\subsection{Synthesis of copolymer I1-B1}

Polymerization was carried out using the method of radiation initiation, for which benzyl methacrylate (BzMA) and isodecyl methacrylate (IDMA) were treated with a beam of accelerated electrons from the ILU-6 accelerator (BINP SB RAS) with the electron energy of $2.4 \mathrm{MeV}$, pulse beam current of $320 \mathrm{~mA}$, pulse duration of $0.6 \mathrm{~ms}$, pulse repetition rate of $2.5 \mathrm{~Hz}$, underbeam transportation velocity of $2 \mathrm{~cm} / \mathrm{s}$. Dose is $20 \mathrm{kGy}(\mathrm{J} / \mathrm{g})$.

After radiation treatment of the monomers, their mixture with a molar ratio of 1:1 was kept at $70-120{ }^{\circ} \mathrm{C}$ for $4-8$ hours. After thermal treatment, the polymer was purified by dissolving it in ethyl acetate and precipitating the polymer with ethyl alcohol. The 
procedure was repeated 3 times. Thereafter, drying was carried out at a temperature of $50^{\circ} \mathrm{C}$ and under reduced pressure for 10 hours. The copolymer yield was at least $80 \%$.

\subsection{Characterization}

The homogeneity of the copolymer I1-B1 prepared at a temperature of $70{ }^{\circ} \mathrm{C}$ (processing time $\sim 24$ hours) was checked by separating fractions by fractional precipitation from an ethyl acetate solution by adding ethanol.

Molecular-weight distribution was determined by means of gel-permeation chromatography (GPC) using a chromatographic system with refractometer detector (Knauer, Germany), column PLgel $20 \mu \mathrm{m}$ MIXED-A, 300 x $7.5 \mathrm{~mm}$ (Agilent), which was calibrated using the standards of PMMA Calibration Kits (Mp 900 - 1500000) (Agilent). The solvent was THF, flow rate $1 \mathrm{ml} / \mathrm{min}$.

At the initial stages of polymerization (for the degree of conversion not more than $5 \%$ ) the ratio of the units in link was determined by means of nuclear magnetic resonance on an AVANCE III 500 NMR spectrometer (Bruker). The solutions in $\mathrm{CDCl}_{3}$ were prepared for investigation.

The study of the dynamic viscosity of polymer solutions was carried out using the solutions in ethyl acetate at temperatures from 25 to $65{ }^{\circ} \mathrm{C}$ on LVDV-II+ (Brookfield, USA).

\section{Results and discussion}

Monomers were processed with a beam of accelerated electrons using an ILU-6 accelerator (BINP SB RAS). After that, the equimolar mixture was subjected to heat treatment at various temperatures. At the initial stages of polymerization (for the degree of conversion not more than 5\%), samples were taken and the polymer was isolated from them. Using the method of ${ }^{13} \mathrm{C}$ NMR spectroscopy in the isolated polymer, the ratio of the units was determined. Fig. 2 shows the temperature dependence of their ratio in the copolymer in Arrhenius coordinates. At temperatures of $70-90{ }^{\circ} \mathrm{C}$, the change in the ratio of the links is linear. Further, a sharp increase in the content of isodecyl methacrylate units in the polymer is observed, and at temperatures of $110-120^{\circ} \mathrm{C}$, isodecyl methacrylate absolutely prevails in the polymer. Such a change in the relative reactivity can be associated with a change in the geometry of isodecyl methacrylate [4]. The optimum temperature for further production of the copolymer was determined as $70^{\circ} \mathrm{C}$.

The copolymer synthesized at $70{ }^{\circ} \mathrm{C}$ was tested for homogeneity using the fractional deposition method. The isolated fractions were studied by IR spectroscopy and gel permeation chromatography (GPC). The results of GPC studies of the initial copolymer and a number of isolated fractions are shown in Fig. 3. The molecular weight of the copolymer is the same as that of the isolated fractions and is $\mathrm{Mw} \sim 1,750,000 \mathrm{~g} / \mathrm{mol}$, the degree of polydispersity is $\mathrm{Mw} / \mathrm{Mn} \sim 2.89$. So, the homogeneity of the synthesized copolymer can be argued. 


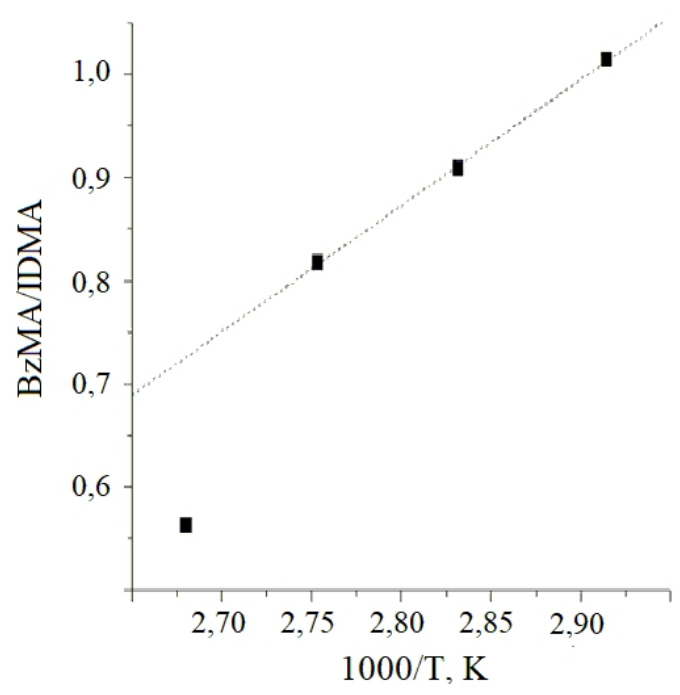

Fig. 2. Temperature correlation of the ratio of monomers in the copolymer.

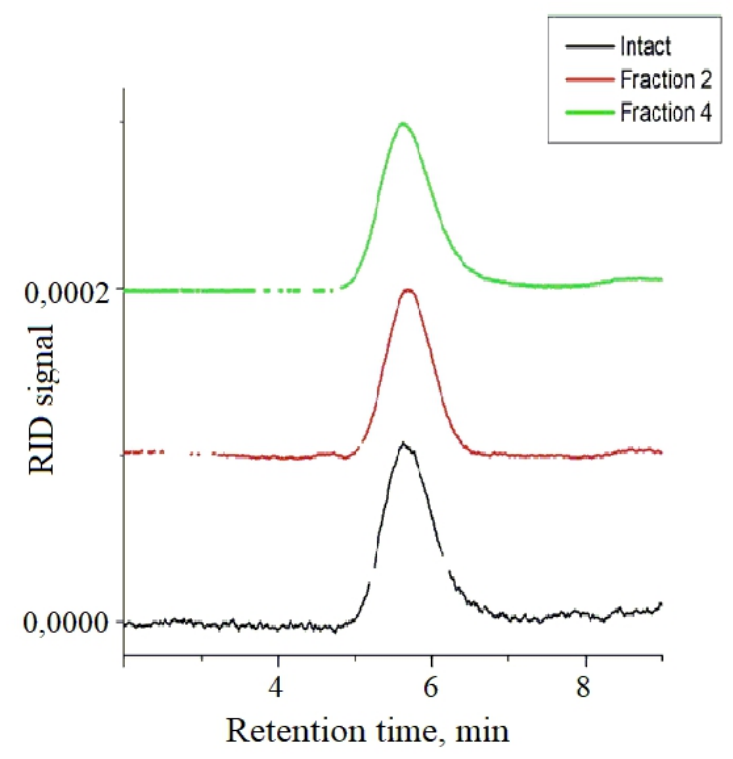

Fig. 3. GPC data on the copolymer and its two fractions. 
The temperature dependence of the viscosity of copolymer solutions in ethyl acetate is shown in Fig. 4. It is atypical for polymers. When heated, the viscosity increases, and then it stabilizes within the experimental error. This behavior usually indicates the presence of a coil-chain transition, similar to that reported by Birshtein et al. [5].

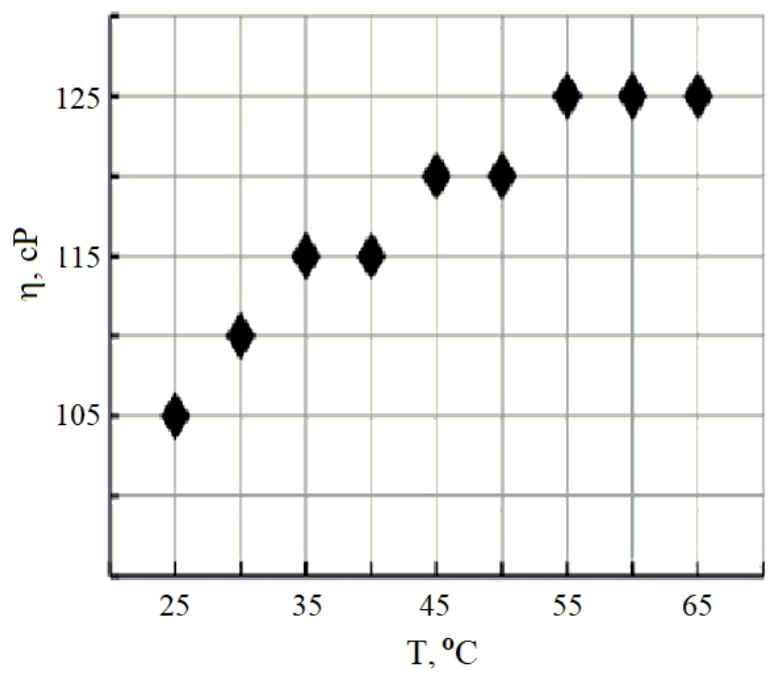

Fig. 4. Temperature correlation of the viscosity of the copolymer.

\section{Conclusions}

The experiments were carried out on the radiation-initiated polymerization of methacrylic acid derivatives with bulky substituents (isodecyl and benzyl). The regularities of its formation are investigated. The conditions for obtaining a homogeneous copolymer are determined. A polymer with the molecular weight of more than $10^{6} \mathrm{~g} / \mathrm{mol}$ was obtained. An increase in the viscosity of the copolymer with an increase in the temperature of the solution was shown.

This work was supported by the Ministry of Education and Science of the Russian Federation (project No. AAAA-A19-119062690004-0).

\section{References}

1. R. Jin, Y. Hanazaki, T. Nishikubo, Polym. J. 29, 113 (1997)

2. W.S. Lyoo, S.K. Noh, J.H. Yeum, G.C. Kang, H.D. Ghim, J. Lee, B.C. Ji, Fibers Polym. 5, 75 (2004)

3. M. Yuan, L. Xu, X. Cui, J. Lv, P. Zhang, H. Tang, Polymers 12, 2747 (2020)

4. N. Ayaz, F. Bezgin, K. Demırell, ISRN Pol. Sci. 352759 (2012)

5. T. M. Birshtein, V. A. Pryamitsyn, Macromolecules 24, 1554 (1991) 